\title{
Foot Digit 1 Phalanx
}

National Cancer Institute

\section{Source}

National Cancer Institute. Foot Digit 1 Phalanx. NCI Thesaurus. Code C52778.

A bone in the largest and most medial toe of the foot. 